\title{
VI. Flüchtlingshilfe
}

Schauff war auf dieser zweiten Reise bei einem Treffen in Düsseldorf, das Karl Arnold und Heinrich Lübke - zu dieser Zeit war Arnold Ministerpräsident und Lübke Landwirtschaftsminister in Nordrhein-Westfalen - organisiert hatten, ebenfalls mit Freunden aus der inneren Kolonisation zusammengekommen, die die Diktatur überlebt hatten. Dabei wurden Fragen der Fortführung dieser Arbeit angesichts der drängenden Flüchtlingsproblematik erörtert wie auch die Möglichkeiten der Migration und Siedlung in Übersee, über die Schauff zu berichten wußte ${ }^{1}$. Eine wichtige Begegnung, die Schauff in seinem Entschluß bestärkte, sich der internationalen Flüchtlingsbewegung zu widmen, war die mit Kardinal Josef Frings in Köln. Der Kardinal nahm regen Anteil an der Flüchtlingsproblematik und sollte die Arbeit Schauffs auf diesem Gebiet auch künftig intensiv begleiten².

\section{Organisation der Flüchtlingshilfe nach 1945}

Das Schicksal von Verfolgung und Flucht, das Johannes Schauff am eigenen Leib erfahren hatte, war nach dem Kriege für ihn der wesentliche Anlaß, den Menschen zu helfen, die durch die nationalsozialistische Diktatur und den Krieg zum menschlichen Treibgut geworden waren. Auf seiner Deutschlandreise konnte er sich durch den Besuch einiger Lager selbst vom Schicksal der Flüchtlinge und dem Los der sogenannten Displaced Persons (DPs) überzeugen.

Schon unmittelbar nach Kriegsende hatte Schauff dem apostolischen Nuntius in Brasilien die Notwendigkeit der Schaffung einer internationalen katholischen Flüchtlingsorganisation dargelegt ${ }^{3}$; über die neu entstandenen Migrations- und Flüchtlingsprobleme war er auch brieflich von seinem politischen Weggefährten und Freund Karl Thieme informiert worden 4 . Während seiner ersten Europareise hatte er dann im Februar 1948 in Rom ein Memorandum zur katholischen Flüchtlingshilfe verfaßt, das durch Monsignore Kaas und Pater Robert Leiber zur

1 Vgl. den Briefwechsel zwischen 1947 und 1949 mit Karl Fütterer, Wilhelm Boyens, Johann H. Haefs, Max Helble und Johann Schäfer (IfZ, NL Schauff, Bd. 32-37).

2 Schauff, Autobiographische Notizen/Fragmente, o. p.; zur Flüchtlings- und Vertriebenenpolitik während der Amtszeit von Kardinal Frings vgl. den entsprechenden Bestand im Historischen Archiv des Erzbistums Köln, CR II (betr. auch die Tätigkeit der ICMC und ihre Zusammenarbeit mit dem deutschen Episkopat, insbesondere mit Kardinal Frings; vgl. dazu auch die Korr. Johannes Schauff mit Prälat Böhler/Katholisches Büro, ebenda CR II, 22, 22 h, 5); Schreiben Johannes Schauff an P. Robert Leiber vom 6. Februar 1950 (IfZ, NL Schauff, Bd. 14).

3 Memorandum vom 20. August 1945 (IfZ, NL Schauff, Bd. 14).

4 Thieme (siehe oben, S. 38 sowie Anm. 72) setzte Schauff über die Vertreibungen aus dem Osten ins Bild und meinte, hier läge für ihn ein neuer Aufgabenbereich (Schreiben Thieme an Schauff vom 12. Februar 1946); Schreiben Schauff an Thieme in der gleichen Angelegenheit vom 18. Juli 1946, zit. S. 94 Anm. 9; beide Schreiben in IfZ, NL Schauff, Bd. 8). 
Kenntnis von Pius XII. gebracht wurde und das wohl als eigentlicher Anstoß für die spätere Gründung der "International Catholic Migration Commission“ (ICMC) gelten kann ${ }^{5}$.

In diesem Memorandum erhebt Schauff die Forderung nach einem "pflichtgemäßen Beitrag" der katholischen Kirche zur Lösung der Flüchtlingsfrage, die angesichts der exorbitanten Zahlen der sich vor allem in Westdeutschland und Österreich befindenden DPs - Schauff sprach von zwölf Millionen - von der "International Refugee Organisation“ (IRO) allein nicht gelöst werden könne. Zudem fehle es an einheitlichem und planmäßigem Handeln, das vor allem angesichts nəuer globaler Bedingungen unabdingbar sei. Darüber hinaus würden Flüchtlingshilfe und Migration zunehmend aufgrund der Einwanderungsrestriktionen außereuropäischer Aufnahmeländer erschwert, die in erster Linie für Deutschland gälten. Hier könne die katholische Kirche vor allem in Lateinamerika ihren Einfluß geltend machen.

Schauffs konkreter Vorschlag lautete, in Genf eine entsprechende „Arbeitsstelle für Flüchtlinge, Auswanderung und Siedlung" zu schaffen. Sie solle auf der einen Seite die Arbeit der verschiedenen bereits bestehenden katholischen Organisationen koordinieren, die bereits auf nationaler und internationaler Ebene Flüchtlingshilfe leisteten ${ }^{6}$, und zum andern ihren Einfluß bei den entsprechenden internationalen Organisationen ${ }^{7}$ geltend machen. Eine solche „internationale Arbeitsstelle" müsse mit einigen ständigen Fachleuten besetzt werden, wobei vor allem Personen mit Erfahrungen in Europa sowie Nord- und Südamerika beizuziehen seien. Daneben müsse ein Beratungskomitee maßgeblicher katholischer Persönlichkeiten aus Politik und großen Sozial- und Wohlfahrtsverbänden gebildet werden. Darüber hinaus gelte es, die Tätigkeit der Arbeitsstelle durch einen Beauftragten des Heiligen Stuhls mit den diplomatischen Aktivitäten des Vatikans abzustimmen.

In diesem Memorandum umriß Schauff zugleich die neue Qualität der Nachkriegsmigration: An die Stelle der spontanen Einzelwanderung sei zunehmend die dirigierte Massenwanderung getreten, an deren Ende nicht mehr der endgültige Ansiedlungsprozeß von Familien stehe, sondern die reine Arbeitskraftvermittlung. Die Verelendung der Nachkriegszeit und das damit verbundene Anwachsen karitativer Tätigkeit berge zudem die Gefahr, den Wanderungs- und Ansiedlungsprozeß, der eine Aufgabe für Pioniere sei, „nicht in erster Linie wirtschaftlich auf

5 Pro-Memoria zur internationalen Zusammenfassung der katholischen Arbeit für Flüchtlinge, Wanderung und Ansiedlung (Februar 1948), IfZ, NL Schauff, Bd. 14.

6 Schauff nennt auf internationaler Ebene die Vertretung des Vatikans bei der IRO, die Internationale Caritas mit ihrer Sektion „Profugi“ sowie die Bemühungen der interamerikanischen katholischen sozialen Aktion (Kongreß in Rio de Janeiro 1948); auf nationaler Ebene die amerikanischen Organisationen National Catholic Welfare Conference (NCWC) und War Relief Services (WRS); in Deutschland der Raphaels-Verein zur Betreuung katholischer Auswanderer im Rahmen des Caritas-Verbandes; in Italien und dem Vatikan die Giunta Cattolica di Emigrazione und Pontificia Commissione Assistenza sowie in den verschiedenen südamerikanischen Ländern die Einwanderungsabteilungen der Acción Social Cattolica (ASA).

7 Hier nennt Schauff neben der IRO das Internationale Arbeitsamt (International Labour Organisation - ILO) sowie die Organisation des Marshall-Plans. 
der Basis der Selbstverantwortung durchzuführen, sondern ihn durch zu starke sozial-fürsorgerische Gesichtspunkte seiner Kraft zu berauben."

Auf diese Denkschrift erfolgte von seiten des Vatikans zunächst keine Reaktion. Am 27. November 1949 klagte Schauff in einem Brief an den Jesuitenpater Robert Leiber in Rom, daß sich in der Angelegenheit einer katholischen Migrationsorganisation nichts bewege. Leiber, langjähriger Berater und persönlicher Vertrauter von Pius XII. ${ }^{8}$, nahm daraufhin die Dinge in die Hand, zumal der zuständige Substitut im vatikanischen Staatssekretariat, Montini - der spätere Papst Paul VI. -, erkrankt war.

Montini war ursprünglich ein Gegner einer autonomen katholischen Flüchtlingsorganisation, die er allenfalls direkt an Rom anbinden und kontrollieren wollte; hinzu kam, daß bereits ein vatikanisches Migrationsbüro als Verbindungsstelle zur IRO in Genf bestand. Doch obsiegte der Einfluß Leibers, der sich Schauffs Position, eine unabhängige Organisation zu gründen, zu eigen gemacht hatte ${ }^{9}$. Auch Montini nahm schließlich eine konstruktive Haltung zur Gründung der späteren ICMC ein ${ }^{10}$.

Schauff seinerseits warb vor allem in Deutschland und Brasilien für das projektierte Unternehmen und drängte angesichts der internationalen Entwicklung der Flüchtlingspolitik zur Eile. Da Brasilien eines der wichtigsten Aufnahmeländer für eine solche Migration darstellte, unterrichtete er in Rio de Janeiro unmittelbar den vatikanischen Nuntius, und dort traf er auch mit Monsignore Luigi Ligutti zusammen, der in Rom im Auftrag des Vatikans als Beobachter bei der Food and Agriculture Organisation (FAO) akkreditiert war, um über das weitere Procedere zu diskutieren ${ }^{11}$.

In Deutschland bestanden wichtige Verbindungen zu Oswald von Nell-Breuning, vor allem aber weiterhin zu Kardinal Frings, der als deutschen Vertrauensmann für das geplante Migrationskomitee den bundesdeutschen Vertriebenenminister Hans Lukaschek ${ }^{12}$ benannte und anläßlich einer Romreise als Vorsitzender der Fuldaer Bischofskonferenz im Vatikan eine Note betreffend die Errichtung einer internationalen katholischen Flüchtlingsorganisation überreichte ${ }^{13}$. In der weiteren Personaldiskussion schlug Schauff Professor Josef Haltmayer als Vertreter für die in Österreich befindlichen Flüchtlinge, vor allem die Donauschwaben,

8 Robert Leiber (1887-1967), 1906 Eintritt in den Jesuiten-Orden, seit 1924 enger Mitarbeiter des damaligen päpstlichen Nuntius Eugenio Pacelli, vermittelte während des Zweiten Weltkrieges Kontakte zwischen dem Heiligen Stuhl und verschiedenen Widerstandsgruppen. Lehrte als Kirchenhistoriker ab 1930 an der Gregoriana in Rom. Vgl. Karl H. Neufeld, Robert Leiber, NDB Bd. 14; Deutsche Biographische Enzyklopädie, Bd. 6, München u. a. 1997, S. 302.

9 Korrespondenz Schauff-Leiber vor allem 1951/52 (IfZ, NL Schauff, Bd. 14).

$10 \mathrm{Vgl}$. die Korrespondenz u.a. zwischen Schauff und Ligutti, Norris sowie Erzbischof Francis B. Keough, Baltimore 1950/51 (IfZ, NL Schauff, Bd. 12 u. 15).

$11 \mathrm{Vgl}$. Vincent A. Yzermans, The people I love, S. $119 \mathrm{ff}$.

12 Hans Lukaschek, ehemaliger Zentrumspolitiker und Oberpräsident der preußischen Provinz Oberschlesien, gehörte während des Krieges zum „Kreisauer Kreis“ (vgl. Mehringer, Widerstand und Emigration, S. $191 \mathrm{ff}$.).

13 Schauff an Leiber, 27. Februar 1950; Schauff an Msgr. Ligutti, 27. Februar 1950 (IfZ, NL Schauff, Bd. 14 und 15). 
vor $^{14}$. In Rom selbst erreichte Leiber schließlich einen Durchbruch im Sinne Schauffs, wobei beider Haltung insbesondere durch den Jesuiten Gustav Gundlach unterstützt wurde, der wie Leiber an der päpstlichen Universität Gregoriana lehrte, sowie nicht zuletzt durch Ludwig Kaas.

Mit dem Plazet des Papstes formulierte Leiber in Schreiben an Kardinal Frings und an Schauff zentrale Punkte, die von Schauffs Vorstellungen ausgingen und später für die ICMC konstitutiv wurden: Leitung durch Personen, die auf dem Gebiet der Migration und Siedlung Erfahrung hätten; Ausrichtung der Arbeit nach wirtschaftlich-sozialen und nicht nach karitativen Gesichtspunkten; die Kommission solle keine päpstliche Kommission, wohl aber vom Heiligen Stuhl anerkannt und mit ihm organisch verbunden sein ${ }^{15}$.

$\mathrm{Zu}$ diesem Zeitpunkt war von vatikanischer Seite bereits die Entscheidung über die personelle Besetzung der Führungsspitze der Migrationsorganisation gefallen. Die Wahl fiel auf jene drei Männer, die bereits während Schauffs erster Nachkriegsreise nach Europa 1947 in Rom zusammengekommen waren und damals bereits, angeregt von Pius XII., das Flüchtlingsproblem thematisiert hatten: James J. Norris, Luigi Ligutti und Johannes Schauff. Schauffs Drängen auf eine katholische Initiative bei der Lösung der internationalen Flüchtlingsproblematik hing eng mit der sich nunmehr abzeichnenden Politik der Westmächte, vor allem der Vereinigten Staaten zusammen, wobei Schauff auch entsprechende Aktivitäten der Evangelischen Kirche vor Augen hatte ${ }^{16}$. Angesichts dieser internationalen Entwicklung war Schauff bemüht, auch die unterdessen konstituierte Bundesrepublik Deutschland in die Pflicht zu nehmen. Mit Unterstützung von Heinrich Lübke und Hermann Kopf propagierte er aufs neue den Gedanken der Auslandssiedlung und versuchte mit einem eigens gefertigten Memorandum den Bundeskanzler zu gewinnen 17: Die Zielgruppe derjenigen, deren Migration unterstützt werden sollte, umfaßt Heimatvertriebene aus dem deutschen Osten und Volksdeutsche aus Südosteuropa, die nur schwer in den jungen deutschen Weststaat zu integrieren seien, aber auch Landarbeiter aus Italien. Es sollte sich um homogene Gruppen und Landsmannschaften möglichst bäuerlicher Provenienz handeln, für die

14 Vgl. das Schreiben Schauff an Andreas Rohracher, Erzbischof von Salzburg, vom 18. 3. 1950 betr. die Gründung und personelle Zusammensetzung der ICMC (IfZ, NL Schauff, Bd. 14).

15 Schreiben Leiber an Schauff vom 15. 3. 1950 sowie Leiber an Kardinal Frings vom 9. 3. 1950, IfZ, NL Schauff, Bd. 14.

16 Vgl. Schauffs Hinweise auf den sogenannten Walter-Bericht im amerikanischen Repräsentantenhaus über "Vertriebene und Flüchtlinge volksdeutschen Ursprungs“ (Bericht Nr. 1841, 81. Kongreß, 2. Sitzungsperiode, 24. 3. 1950) sowie auf die Resolution der drei westlichen Außenminister auf der Londoner Konferenz vom 14. 5. 1950 zum Problem des Bevölkerungsüberschusses in den Ländern Westeuropas. Im Zusammenhang mit den protestantischen Aktivitäten bemerkt er, daß er immer wieder auf die Figur des Dr. Gerstenmaier gestoßen sei, „der überall, zwar nicht die obersten sichtbaren, aber doch die arbeitsmäßigen Positionen besetzt hat" (Brief an Pater Leiber vom 6. 2. 1950, IfZ, NL Schauff, Bd. 14).

17 „Europäische Überseesiedlung. Bemerkungen zur deutschen Auswanderungsfrage“, Juni 1950 (IfZ, NL Schauff, Bd. 14); vgl. auch den entsprechenden Briefwechsel mit dem Ministerialdirigenten im Bundeskanzleramt, Herbert Blankenhorn, ebenda. 
vor allem in den lateinamerikanischen Ländern eine hohe Akzeptanz gegeben sei. Bei einem solchen „landwirtschaftlich-ländlichen Umsiedlungsprozeß “ müßten die Aufnahmeländer einen sozialen und ökonomischen Zugewinn erwarten dürfen. Um die Organisation der Auswanderung sollten sich deutsche staatliche Stellen im Verbund mit anderen europäischen Staaten kümmern - insbesondere mit Frankreich, Österreich und Italien.

Schließlich trat das Vorhaben in die Phase konkreter Umsetzung ein: Im September 1950 trafen sich in dem römischen Restaurant Ranieri die Vertreter von 14 freien katholischen Verbänden zur Vorbereitung der Gründung einer internationalen Flüchtlingshilfe- und Migrations-Organisation ${ }^{18}$. Von dieser Versammlung erging an Schauff, Norris und den Italiener Giovanni Battista Vicentini - als Vertreter der Organisationen seines Landes - der Auftrag, einen provisorischen Statutenentwurf zu erarbeiten. Dies geschah Anfang Oktober 1950 in Padua. Der Entwurf, den Schauff an Leiber zur Begutachtung weiterleitete, war eine Überarbeitung der Gedanken, die er sich bisher zu diesen Fragen gemacht hatte; er bildete die Grundlage für die spätere definitive Satzung. Das provisorische Statut wurde schließlich von dem zuständigen vatikanischen Staatssekretär, Montini, als Arbeitsgrundlage „approbiert" 19 .

Zusammengefaßt ergaben sich folgende zentralen Punkte: Die Organisation sollte durch einen vom Vatikan benannten kirchlichen Verbindungsmann mit dem Heiligen Stuhl verbunden sein, der sich auch das Agrément bei der Benennung des Präsidenten vorbehielt. Der organisatorische Aufbau gliederte sich in einen Rat mit 15 Mitgliedern, ein dreiköpfiges sogenanntes Regierungskomitee und die $\mathrm{Ge}-$ neralversammlung. Daneben wurden das Verwaltungsamt eines Generalsekretärs und ein Informationsbüro geschaffen. Als Ratsmitglieder sollten von den Bischöfen besonders kompetente Fachleute in Sachen Migration ernannt werden.

Dieses provisorische Statut wurde vom Papst als Grundlage für die Tätigkeit der „International Catholic Migration Commission“ (ICMC) anerkannt ${ }^{20}$. Daraufhin erfolgte mit dem ersten Treffen des ICMC-Rates am 5. Juni 1951 in Rom der entscheidende Schritt zur tatsächlichen Schaffung der neuen internationalen Flüchtlingshilfe-Organisation. Bei dieser Tagung, an der auch Montini teilnahm, waren mit Australien, Kanada, Argentinien und Brasilien sowie den Vereinigten

18 Anwesend waren Msgr. Joseph McGeough als Vertreter des Staatssekretariats; Edward E. Swanstrom, Weihbischof von New York, und Andrew Landi als Vertreter der NCWC Relief Services; Msgr. Crivelli von der Caritas Internationalis, Luzern; Pater Froehling vom St. Raphaels-Verein, Hamburg; Msgr. John O'Grady von der amerikanischen Caritas (in Vertretung von Msgr. Ligutti); Pater Edward J. Killion vom vatikanischen MigrationsVerbindungsbüro in Genf sowie Norris, Schauff und Vicentini.

19 Schreiben Montini an James J. Norris, Acting Chairman ICMC, Genf, vom 12.4. 1951 (mit Anhang „Provisional Constitution of the International Catholic Migration Commission" mit Korrekturen Schauffs), IfZ, NL Schauff, Bd. 12.

$20 \mathrm{Vgl}$. das entsprechende Schreiben Montini an den "acting chairman“ Norris vom 12.4. 1951, in dem noch einmal nachfolgende Punkte präzisiert werden: 1) Die ICMC solle eng mit dem Heiligen Stuhl zusammenarbeiten; 2) Anderungen der Statuten und Ernennung des „chairmans“, der Ratsmitglieder und des Generalsekretärs sollten vom Heiligen Stuhl approbiert werden; 3) die Sitzungsprotokolle sollten vor der Veröffentlichung vorgelegt werden (IfZ, NL Schauff, Bd. 12). 
Staaten fünf der überseeischen Immigrationsländer vertreten, Europa durch Italien, die Bundesrepublik Deutschland, die Niederlande und Großbritannien. Schauff wurde durch Montini „ad personam“ in den fünfzehn Mitglieder umfassenden Rat berufen und zugleich Vizepräsident der ICMC. Als Generalsekretär fungierte der Kanadier John Lanctot. Die Herausgabe einer Zeitschrift, die regelmäßig erscheinen und über die Tätigkeit der nationalen Kommissionen sowie über aktuelle Probleme der Migration berichten sollte, wurde ebenso beschlossen wie eine geographische Aufteilung der Operationsfelder: Schauff übernahm die Verantwortung für Lateinamerika, während Norris die Migration nach Nordamerika vorbereiten sollte. Die Finanzierung des Unternehmens erfolgte zunächst durch die amerikanische National Catholic Welfare Conference (NCWC), aus der der amtierende Präsident der ICMC, James J. Norris, hervorgegangen war ${ }^{21}$. Sitz der neuen Organisation wurde Genf; das bisher dort bestehende vatikanische Verbindungsbüro unter Pater Killion wurde aufgelöst ${ }^{22}$.

Am 24. Januar 1952 schließlich wurde das Zentralbüro der ICMC in Genf eröffnet, und im Oktober 1952 erfolgte Schauffs erste offizielle Mission als Vizepräsident der ICMC nach Lateinamerika, an der auch Paulus Gordan und Francesco Cantuti von der italienischen Flüchtlingsorganisation teilnahmen. Schauff besuchte Brasilien, Argentinien, Chile sowie später auch Venezuela, Kolumbien und Peru'23. Auf dieser Reise stellte er erste Kontakte zwischen Kirche und zivilen Behörden her und konnte auch ein erstes Mitarbeiternetz installieren.

Die Reise diente auch dazu, in diesen Ländern das Mißtrauen gegenüber dem durch die NS-Verbrechen stigmatisierten Deutschland abzubauen. Bereits in seinen frühen Memoranden hatte Schauff darauf gedrängt, eine „politische Entgiftung“ durchzuführen und auf eine „Entnazifizierung der Reste des sogenannten Auslandsdeutschtums hinzuarbeiten, wobei durchaus auch Träger der katholischen deutschen Auslandsseelsorge auf den Prüfstand kommen sollten" 24 .

In der Frage der „Entnazifizierung“ der deutschen Migration stimmte Schauff vor allem mit Leiber überein, der seinerseits bemüht war, in Lateinamerika unbelastete Mitglieder insbesondere aus seinem Orden zur Unterstützung der ICMC zu gewinnen. Aufgrund seiner eigenen Erfahrung vermochte Schauff bei seinen lateinamerikanischen Gesprächspartnern aber auch auf jenes „andere Deutschland“ hinzuweisen, das eine kollektive Schuldzuweisung nicht möglich mache.

Schauff hielt sich in den darauf folgenden Jahren häufig in Lateinamerika auf ${ }^{25}$. Bereits 1952 wurde das erste Büro in Buenos Aires eingerichtet, das später den Namen „Comisión Católica Argentina de Imigración“ erhielt. Dieses Büro betreute vor allem jugoslawische und italienische Immigranten. In den ersten zwei Jahren wurden 600 Visa für Flüchtlinge bereitgestellt und sogar ein „Tag des Ein-

21 Norris wurde vom Chef der NCWC, dem New Yorker Bischof Swanstrom, im September 1950 für seine Tätigkeit in der ICMC beurlaubt.

22 Schreiben Montini an Norris vom 16. 8. 1951 (IfZ, NL Schauff, Bd. 12).

23 Vgl. Schauff, The ICMC Mission to Latin America, October 1952-January 1953. Summary Report presented to the Governing Committee of the International Catholic Migration Commission, Vipiteno (Sterzing)/Genf 1953 (IfZ, NL Schauff, Bd. 15).

24 Memorandum an Pius XII. vom Februar 1948 (s. S. 99 f., Anm. 5).

25 Reisedispositionen und -korrespondenz bis 1955 in IfZ, NL Schauff, Bd. 12. 
wanderers" (Jornada de la Imigración“) eingeführt ${ }^{26}$. Im September 1952 wurde in Rio de Janeiro in Brasilien ein weiteres Büro eröffnet, das eng mit der Einwanderungskommission des brasilianischen Episkopats („Comissão Nacional Católica de Imigração “) unter der Präsidentschaft des damaligen Weihbischofs von Rio, Hélder Câmara, zusammenarbeitete. In Venezuela wurde im März 1953 mit Unterstützung von Stefan Falez, dem späteren Generalsekretär der ICMC, ein Büro eingerichtet; später erfolgte die Gründung der „Comisión Católica Venezolana de Migración“. In Chile hatte Christoph von Unterrichter den Boden für ein Engagement der ICMC bereitet; unter seiner Leitung wurde im April 1953 ein Stützpunkt in Santiago de Chile eingerichtet ${ }^{27}$. In Kolumbien, bis 1954 unter der Jurisdiktion von Venezuela, wurde ebenfalls ein "Comité Católico Colombiani de Imigración“ unter der Leitung des Litauers Stany Sirutis gebildet ${ }^{28}$. Schließlich vereinigten sich später andere nationale Einwanderungskommissionen wie die von Paraguay und Peru mit der ICMC.

Voraussetzung für den Erfolg dieser Unternehmungen war allerdings eine dauerhafte Finanzierung, die eng mit der internationalen Anerkennung der ICMC zusammenhing. 1951 war die ICMC durch ihren Generalsekretär erstmals bei den Verhandlungen der UNO vertreten, die in die Genfer Flüchtlingskonvention vom 26. Juli 1951 mündeten. Auf dieser Konferenz wurden offizielle Verbindungen zur IRO hergestellt, die den Weg zur Finanzierung auch der ICMC ebneten. Nachdem die amerikanische NCWC - unter Beteiligung der Ford Foundation über die ersten Hürden hinweggeholfen hatte ${ }^{29}$, wurde das Budget der ICMC durch Mittel aufgestockt, die das 1951 gegründete Intergovernmental Committee for European Migration (ICEM) zur Verfügung stellte. Diese finanzielle Grundlage erlaubte es, 1952 einen eigenen "International Catholic Migrant Loan Fund“ (ICMLF) einzurichten, dessen Sekretär Msgr. Andrea Landi wurde; als Treuhänder der ICMC fungierten Johannes Schauff und Giovanni Battista Vicentini.

Mit diesem Fonds, der sich bis Mitte der fünfziger Jahre bereits auf über drei Millionen Dollar belief ${ }^{30}$, konnte die Auswanderung unter der Voraussetzung der Rückzahlung finanziert werden. Die den Auswanderern zur Verfügung gestellten Beträge waren zinslos und hatten lange Rückzahlungsfristen. Zum einen konnte man damit verhindern, daß die Betroffenen durch profitorientierte Agenturen ausgenützt wurden, zum andern war dieses Procedere - und dies war vor allem

$26 \mathrm{Zu}$ diesen ersten organisatorischen Leistungen der ICMC und von Schauff in Lateinamerika vgl. Tadeusz Stark, Die Pionierjahre der Internationalen Katholischen Auswanderungskommission, in: Um der Freiheit willen, S. $210 \mathrm{ff}$.

27 Im ersten Jahre des Bestehens des Büros wurden 750 Visa erteilt und 701 Flüchtlinge und Staatenlose untergebracht. Der ausführliche Briefwechsel Unterrichter-Schauff (19511987) in IfZ, NL Schauff, Bd. 13 (mit einem biographischen Anhang zu Christoph von Unterrichter).

28 Korrespondenz in IfZ, NL Schauff, Bd. 12.

29 Minutes of the Governing Committee meeting, Rom, 5. 4. 1954; Report of the ViceChairman (Schauff) to the Fifth Council Meeting, 14.-15. 9. 1955, Teil III (IfZ, NL Schauff, Bd. 15).

30 ICMC Movements 1952 to June 1955/ICMC Volags (Voluntary Agencies) Revolving Fund Movements, 6. 9. 1955, NL Schauff, Bd. 15 (dort genaue Angabe der Gesamtsumme mit 3705200 Dollar). 
Schauffs Credo - geeignet, die Eigeninitiative und das Selbstwertgefühl der Betroffenen zu stärken ${ }^{31}$. Und wirklich versetzte der über Jahre hinweg stetige Rückfluß dieser Kredite die ICMC in die Lage, Flüchtlingen beim Transport und den ersten Ansiedlungsschwierigkeiten zu helfen.

Es bestand eine Arbeitsteilung mit dem „Intergovernmental Committee for European Migration“ (ICEM), das in erster Linie auf Regierungsebene agierte. Dessen Partner für die Durchführung der praktischen Arbeit waren die großen internationalen freien Wohlfahrtsverbände der Katholiken, Protestanten, die jüdischen Verbände, der Weltkirchenrat und die Quäker. Die Aufgabenstellung der ICMC war die Information und Beratung der Migranten sowie der Aufbau eines Netzwerks von nationalen Organisationen in den Auswanderungs- und Immigrationsländern und deren Koordination. In Deutschland waren dies der Caritasverband, der St. Raphaels-Verein und die Flüchtlingsverbände sowie das Vertriebenenministerium. Schauffs europäischer Verantwortungsbereich lag in der Bundesrepublik und in Österreich. Bis 1955, als die Migrationswelle der Nachkriegszeit allmählich abebbte, waren von der ICMC in Verbindung mit der NCWC knapp 125000 Menschen vor allem nach Nord- und Lateinamerika gebracht worden 32 .

In der positiven Zusammenarbeit der ICMC mit den großen internationalen Flüchtlingsorganisationen, allen voran der $\mathrm{UNO}^{33}$ und denen der Europäer, wurde aber zugleich Schauffs moralisches Engagement für die ihm gestellte Aufgabe deutlich. Anläßlich der Nachfolgediskussion um die Stellung des verstorbenen Direktors des ICEM, Gibson, beklagte Schauff das „Bild des zynischen, routinierten internationalen Beamten, dem es um Macht und Stellung, nicht aber um Menschen geht“34. Der Grundsatz der „Wahrung des Menschlichen“ im Prozeß der Migration blieb bei Schauff jedoch immer verbunden mit der Zielvorstellung der individuellen bäuerlichen Siedlung. Dahinter standen sowohl die Erfahrung des Siedlungspolitikers in der Weimarer Republik als auch die eigene Kolonisationserfahrung im brasilianischen Urwald.

Schon in den Exiljahren hatte Schauff mehrmals Gedanken über die Bedingungen und Grundsätze der Migration und Siedlung zu Papier gebracht. Jetzt, 1953, stellt er eine „Strukturveränderung auf der Aus- und auf der Einwanderungsseite“ fest, die bewirke, daß landwirtschaftlichen Kolonisationsprojekten in den überseeischen Ländern Priorität eingeräumt werde. Es sei festzustellen, daß sich die

31 Schauff, Vermerk über die „Verwendung des Eigenkapitals der Auswanderer“, Rom, 6. 6. 1953; Selbsthilfeprogramm für katholische Einzelauswanderer (nationale Staatsangehörige) aus Europa für das Jahr 1954 unter der Leitung der ICMC (Rio Grande del Sul, Brasilien), Genf, 12.12.1953 (IfZ, NL Schauff, Bd. 11).

32 Davon gingen $55,4 \%$ nach Nordamerika (hier vor allem nach Kanada) und 34,3\% nach Lateinamerika. Vgl. Results of the ICMC-Activities in the first half of 1955, compared with the years 1952, 1953 and 1954 (Revolving Fund Movements with ICMC and NCWC submitted to the Council of Members on March 5th, 1955, IfZ, NL Schauff, Bd. 15).

33 Die 1946 gegründete IRO wurde 1952 durch das Hohe Kommissariat der UN für Flüchtlinge mit Hauptsitz in Genf und Vertretungen in den einzelnen Ländern ersetzt.

34 Es geht um die Kandidatur des bisherigen stellvertretenden Chefs der ICEM, Pierre Jacobsen, und um ein Netzwerk - wie Schauff es sieht - von Freimaurern in UNRRA und IRO. Schreiben Schauff an Pater Leiber vom 5. 2. 1955 (IfZ, NL Schauff, Bd. 14). 
Zweckbestimmung der Einwanderung von der Industrialisierung weg und auf eine Erhöhung der landwirtschaftlichen Produktion und die Erschließung von Neulandgebieten bewege. Dabei hätten vor allem Deutschland und Österreich, aber auch Italien ein besonderes Interesse daran, Bauernfamilien in Übersee wieder zu einer Existenz zu verhelfen, die das eigene Land nicht mehr bieten könne ${ }^{35}$.

Schwerpunkte agrarischer Migration in dieser Zeit waren Chile und Brasilien. $\mathrm{Da}$ in Chile mit dem ICMC-Residenten Christoph von Unterrichter ein hervorragender Agrarfachmann tätig war, konnte sich Schauff persönlich vor allem um Brasilien kümmern und diese Tätigkeit mit der notwendigen Oberaufsicht über seinen eigenen Besitz verbinden. Dies war dennoch eine oft drückende Doppelbelastung, die allerdings - die Familie befand sich ab 1951 wieder ständig in Europa durch Karin Schauffs häufige und manchmal längere Aufenthalte auf der Facenda gemildert wurde.

Schauff verstand seine Arbeit für die ICMC als Fortführung seines seit der Weimarer Republik bestehenden Engagements für die Schaffung christlichen Bauerntums, dem in den Immigrationsländern und vor allem in Lateinamerika eine ganz neue Bedeutung zuwachse. „Siedlung“ - so stellt er kategorisch fest - „ist Schaffen eines neuen Bauerntums. Sie ist der ewige Verjüngungs- und Expansionsprozeß lebendigen Landvolks.“ 36 In Lateinamerika gelte es vor allem, „kollektivistischen Großexperimenten“ kommunistischer Provenienz entgegenzutreten. „Die unausgeglichene Agrar- und Eigentumsstruktur mit dem Fehlen eines Mittelstandes, die zunehmende Anteilnahme der Analphabeten und der Einfluß der farbigen Bevölkerung bei ständigem Rückgang der alten portugiesischen und spanischen konservativen Schichten und dazu die rapide Bevölkerungsvermehrung und schnelle ,frühkapitalistische Entwicklung', neben modernsten staatlichen Sozialexperimenten machen manche Staaten in dem Kontinent anfällig für ein kollektivistisches Großexperiment. Der nicht zu leugnende rasche industrielle Aufschwung der Sowjetunion von einem Agrarland mit 80 Prozent Analphabeten zu dem heute zweitgrößten Industriestaat verfehlt deshalb noch mehr als beim Stadt- und Landproletariat bei den Intellektuellen seinen Eindruck nicht, abgesehen von der direkten und indirekten Propaganda Moskaus selbst. Neuerdings übt auch das Beispiel Rotchina eine besondere Attraktion aus ... Wie ist von einer feudalen und halbfeudalen Besitzstruktur der Weg zu einer weiten Streuung des Bodeneigentums zu bahnen und eine vermehrte landwirtschaftliche Produktion und dann eine bessere Ernährung zu erreichen ohne in kollektivistische Sozialexperimente zu verfallen? Autoritäre oder gar kommunistische Regime haben eins voraus: Sie zwingen die oft passive Bevölkerung zu eigenen Anstrengungen und stärkerem Einsatz. Eine freiheitliche Ordnung kann dies aber durch Erziehung, Überzeugung und den Anreiz individueller materieller Kompensation erreichen. Dieser Weg ist viel mühsamer. Ohne die Unterstützung gerade von Europa und seinen geistigen Kräften her kann er kaum zum Erfolg führen." 37

35 Schauff, Memorandum betr. [die] Kolonisation in Übersee, Rom, 15. 6. 1953 (IfZ, NL Schauff, Bd. 11).

36 Schauff, Innere Kolonisation (April 1956), Exzerpt in IfZ, NL Schauff, Bd. 39.

37 Schauff, Die landwirtschaftliche Erschließung, S. 9 f. 
Als Repräsentant der ICMC veröffentlichte Schauff mehrere Memoranden zum Problem der Ansiedlung, darunter zwei Sammelbände des „ICMC Migration Digest": „Die Landsiedlung der Einwanderer in Lateinamerika“ (1955) und „Die Einwandererkolonisation in Brasilien“ (1956). 1957 erschien in deutscher Sprache die von ihm herausgegebene Studie "25 Jahre Rolândia“ und 1959 eine weitere Gemeinschaftsarbeit „Landerschließung und Kolonisation in Lateinamerika". Der Pflege der Öffentlichkeitsarbeit galt Schauffs besonderes Augenmerk. Ab Oktober 1952 gab es die monatlichen "ICMC-News“, deren Leitung ab der 13. Nummer (November 1953) Johannes Schauff selbst übernahm. In Rom erschien gleichzeitig das ICMC-Bulletin, später umbenannt in „Migration News“. Auch bei diesem Bulletin, das die allgemeinen Migrationsprobleme unter sozialen, wirtschaftlichen und rechtlichen Aspekten analysierte, übernahm Schauff die Chefredaktion, später, ab 1956/57, fungierte er als „Editorial Adviser“.

Neben seinen Verpflichtungen als Vizepräsident übernahm Schauff im Januar 1954 zunächst interimistisch auch das Generalsekretariat der ICMC ${ }^{38}$, dem er eine effizientere Struktur zu geben bemüht war. Dies betraf vor allem die Stelle eines ständigen Assistenten des Generalsekretärs für einen Apparat von immerhin sechs Referenten und neun Sekretärinnen bzw. Sekretären sowie fünf Zweigstellenleitern für Lateinamerika. Angesichts der zu bewältigenden inhaltlichen Aufgaben war Schauffs Forderung nach besserer personeller Ausstattung der Leitung des Generalsekretariats durchaus berechtigt: Es ging einmal um eine „Koordinierung der nationalen Wanderungstätigkeit in den Aus- und Einwanderungsländern ..., um die Leistungsfähigkeit der katholischen Wanderungsarbeit zu steigern“; zum anderen um die "Arbeitsanweisung und Kontrolle der Zweigstellen“ in den Aufnahmeländern und schließlich um die „allgemeine Verwaltung der Buchhaltung der Kommission", die erstaunlicherweise erst 1954 von Rom nach Genf verlegt worden war. „Eine große Rolle spielten die finanziellen Kontrollen der Zweigstellen“ sowie die „Statistiken über die Revolving-Fund-Auswanderung und Abrechnungen mit ICEM über die von den Nationalstellen der ICMC durchgeführten Umsiedlungen“; hinzu kam die „Kontrolle und Abrechnung über verschiedene für die Arbeit der ICMC verwandte Fonds" 39 .

Schauff war bei dieser internationalen Aufgabe offenbar in seinem Element. In seine Zeit als Generalsekretär - er übte dieses Amt bis Juli 1956 aus - fallen gewichtige Entscheidungen wie die Installierung der „Konferenz der nationalen Direktoren" als beratender Körperschaft, die am 3. August 1954 erstmals in Genf zusammentrat. Die Revision der ursprünglich ja provisorischen Statuten der ICMC mündete im Oktober 1956 in eine neue Verfassung. In diese Phase fallen

38 Der erste Generalsekretär Lanctot war im September 1953 durch den Benediktiner Paulus Gordan als ,acting general secretary “ abgelöst worden. Gordan, im brasilianischen Exil zeitweise Privatlehrer der Schauffschen Kinder auf der Fazenda, war ein enger Vertrauter und Protégé der Familie Schauff; siehe oben, S. 81 sowie Karin Schauff, Wechselvolles Familienschicksal, S. 27.

39 Schauff erhielt nur für kurze Zeit den geforderten Assistenten. Bericht des Vize-Präsidenten (Schauff) an die Mitarbeiter des Rates über die Tätigkeit des Generalsekretariats der Internationalen Katholischen Kommission für Wanderungsfragen, (Juni) 1955; Minutes Sixth Session ICMC Council (Private Session), 12. 10. 1956 (IfZ, NL Schauff, Bd. 15). 
auch Schauffs erfolgreiche Bemühungen um eine geordnete Finanzbuchhaltung, die jedoch durch chronische Personalknappheit deutlich erschwert wurden ${ }^{40}$.

Neben der Umgestaltung der inneren Organisation bemühte sich Schauff um eine Reform der italienischen Flüchtlingspolitik, deren Versagen er auf die staatliche zentralistisch-bürokratische Gängelung der freien Verbände und Hilfsorganisationen zurückführte ${ }^{41}$. Angesichts von etwa 50000 Müttern und Kindern, die in Italien zurückgeblieben waren, während die Familienväter sich bereits im Auswanderungsland Argentinien befanden, drängte Schauff Pater Leiber, den Vatikan $\mathrm{zu}$ einer Intervention $\mathrm{zu}$ bewegen ${ }^{42}$. Schauff offerierte die Hilfestellung der ICMC beim Aufbau einer effektiven katholischen Fachorganisation für Auswanderung, nachdem die "Giunta Cattolica Italiana per l'Emigrazione“ dieser Aufgabe offenbar nicht gerecht werden konnte ${ }^{43}$ - ein letzen Endes vergebliches Bemühen.

Ein erfolgreicheres Unternehmen war die Ansiedlung donauschwäbischer Flüchtlingsfamilien in Südfrankreich, die von der ICMC in Zusammenarbeit mit der französischen „Secours Catholique“ betreut und von der Ford Foundation finanziert wurde. In der schon erwähnten Zusammenarbeit mit dem Vertreter der Ford Foundation in Österreich, Haltmayer, waren zuerst Pläne gereift, diese Vertriebenengruppe in Südamerika anzusiedel ${ }^{44}$. Nun ergab sich die Möglichkeit, in dem von der Landflucht geplagten Südfrankreich Land zu erwerben, ein Siedlungsprojekt, das von dem damaligen französischen Ministerpräsidenten Robert Schuman unterstützt wurde. Es durften 9200 Flüchtlinge einwandern, Bauernfamilien zumeist, deren wohl bekanntester Siedlungsort das wiederaufgebaute Dorf La-Roque-sur-Pernes in der Provence darstellte ${ }^{45}$. In Zusammenarbeit mit Schauff und der ICMC unterstützte dann diese Bevölkerungsgruppe der „Français du Banat" die spätere Ansiedlung ungarischer Flüchtlinge, die nach dem Aufstand von 1956 ins Land kamen ${ }^{46}$.

Schauffs Aktionsschwerpunkt blieb allerdings weiterhin Lateinamerika ${ }^{47}$. Dorthin dirigierte die ICMC auch eine Gruppe von Flüchtlingen aus der Sowjet-

40 Der entsprechende Vermerk, o.D., in IfZ, NL Schauff, Bd. 14.

41 Vgl. Schauffs Memorandum „Das Versagen der Auswanderungspolitik und -praxis Italiens“, 27. 9. 1953 (IfZ, NL Schauff, Bd. 12).

42 Schreiben an Pater Leiber vom 27. 9. 1953 (IfZ, NL Schauff, Bd. 14).

43 Stefan Falez (Nachfolger Schauffs im Generalsekretariat), „Comprehensive Report on the Development Program“ (1955) betr. Schwierigkeiten der Führung der GCIE (IfZ, NL Schauff, Bd. 12).

44 Schauff, Immigração Colonisadore, o. D. (IfZ, NL Schauff, Bd. 14).

45 IfZ, NL Schauff, Bd. 15; Lamesfeld, Von Österreich nach Frankreich; Lamesfeld, La Roque sur Pernes; Senz, Die Donauschwaben, S. 148-150.

46 Diese Aktion wurde auch von deutschen Regierungsstellen (Bundesminister Heinrich Lübke) sowie Siedlungsorganisationen (Auslandssiedlung/Johann H. Haefs; Katholischer Siedlungsdienst/Prälat Wosnitzer; Deutscher Caritasverband) unterstützt. Vgl. Korrespondenz Schauff-Lamesfeld (Président des Français du Banat) und Schauff-Haefs (IfZ, NL Schauff, Bd. 3 und 14; der Vorgang Siedlung der ungarischen Flüchtlinge ebenda, Bd. 13).

$47 \mathrm{Vgl}$. Report of the Vice-Chairman to the Council Members on the Activities of the General Secretariat of the ICMC, 3. 9. 1954 (IfZ, NL Schauff, Bd. 15). 
union, die den Weg über Honkong und die Philippinen nahm; Johannes Schauff führte vor Ort die Verhandlungen mit kirchlichen und staatlichen Stellen. Besonders engagierte sich Schauff für die vielen polnischen DPs, die sich in Deutschland und Österreich befanden und nicht in ihr unterdessen von den Kommunisten beherrschtes Land zurückkehren wollten. Schauff hatte während seiner Zeit als Abgeordneter des niederschlesischen Wahlkreises Liegnitz Zugang zu Kreisen des katholischen Polens gefunden. Nach seiner Rückkehr nach Europa traf er in Rom auf den Vertreter der Auslandspolen, Bischof Josef Gawlina; dies war der Beginn engerer persönlicher Kontakte zum polnischen Episkopat, die Schauff später auch für die Verbesserung der deutsch-polnischen Beziehungen nutzen konnte. Von daher war es auch kein Zufall, daß einige der wichtigsten Mitarbeiter Schauffs in der ICMC Polen waren, die aus dem diplomatischen Dienst hervorgegangen waren $^{48}$.

Im Juni 1952 fand in Barcelona der erste ICMC-Kongreß mit Vertretern der internationalen Caritas sowie europäischen und überseeischen Hilfsorganisationen statt $^{49}$ - gleichzeitig mit dem dort ebenfalls tagenden Internationalen Eucharistischen Kongreß, der dazu beitrug, auch den Bekanntheitsgrad der katholischen Flüchtlingsorganisation zu erhöhen. Bereits ein Jahr später verkündete der Papst die Apostolische Konstitution „Exsul Familia“, in der die christlichen Prinzipien der Migrationspolitik dargelegt und Gründung und Tätigkeit der ICMC bekanntgemacht wurden, deren Funktion es sei, das "Werk der bestehenden katholischen Organisationen zu vereinigen und zu koordinieren, um den Beistand für Auswanderer und Flüchtlinge zu garantieren "50.

„Exsul Familia“ war auch der Anlaß für Schauffs Bemühungen, in Abstimmung mit Robert Leiber in mehreren Memoranden das Verhältnis von ICMC und Kurie aufs neue und abschließend zu definieren. Vor allem sollte die ICMC beim Staatssekretariat und nicht bei der Konsistorialkongregation ressortieren ${ }^{51}$. Besonderes Gewicht legte Schauff auf die Forderung nach Unterscheidung zwischen der religiös-seelsorgerischen und den mehr "weltlichen" bzw. "technisch-politischen" Aufgaben der Flüchtlingsorganisation. Beide sollten wie Zahnräder ineinandergreifen, wobei es im Interesse der Kirche liege, der ICMC ,jene Freiheit und Selbständigkeit einzuräumen, die ihr auf ihrem Sachgebiet zukommt “52. Diese arbeitsteiligen Unterscheidungsmerkmale finden sich in dem 1956 verabschiedeten

48 Ein Beispiel ist Tadeusz Stark, ein 1912 geborener Jurist im polnischen Außenministerium. Stark kämpfte während des Krieges in der französischen Armee und war ab 1951 Mitglied des Vatikanischen Migrationsbüros bei der IRO, ab 1952 Leiter des Informationszentrums und 1963-1977 Generalssekretär der ICMC.

49 Auf dem Kongreß waren zahlreiche hohe kirchliche Würdenträger vor allem aus Lateinamerika und Polen vertreten; von deutscher Seite referierte Kardinal Frings. Vgl. Stark, Pionierjahre, in: Um der Freiheit willen, S. 215 f.

50 Zit. nach Stark, ebd., S. 216.

51 Schreiben Schauff an Pater Leiber vom 27. 7. 1956, mit einem Anhang „Zu klärende Fragen, Struktur und Zukunftsarbeit der ICMC betreffend“ (IfZ, NL Schauff, Bd. 14).

52 Schauff, "Gedanken und Vorschläge zu einem Arbeitsprogramm der ICMC“, Vipiteno (Sterzing), 16. 5. 1953 (IfZ, NL Schauff, Bd. 14). 
neuen ICMC-Statut in dieser Klarheit allerdings nicht wieder - ebensowenig wie das von Schauff hartnäckig geforderte Prinzip der Hilfe zur Selbsthilfe.

Gleichwohl trugen Schauffs unermüdliche Bemühungen auch für eine innere Strukturreform der ICMC ihm im Sommer 1956 von vatikanischer Seite die Offerte ein, die Präsidentschaft zu übernehmen. In der Korrespondenz zu diesem Vorgang, an der auch Karin Schauff beteiligt war, wird erstmals deutlich, in welchem Ausmaß Schauff und seine Familie im Lauf der Jahre materielle Opfer für die Flüchtlingshilfe erbrachten. Schauffs zahlreiche Unternehmungen im Amt des Vizepräsidenten finanzierte er weitgehend aus eigener Tasche - trotz einiger privater wirtschaftlicher Rückschläge (u.a. niedrigere Kaffee-Ernten durch Witterungsunbilden). Erst als Schauff vorübergehend als Generalsekretär amtierte, erhielt er eine Aufwandsentschädigung ${ }^{53}$, nach seinem Ausscheiden lediglich ein „Honorarium“ in Höhe von 1000 Dollar $^{54}$. Schauff lehnte die angetragene Präsidentschaft schließlich ab; er wurde im Oktober 1956 zum „Honorary Vice-President" ernannt ${ }^{55}$ - in dieser Funktion amtierte er bis 1963 - und war bis 1972 Mitglied des erweiterten Vorstands der ICMC.

$\mathrm{Zu}$ den von Schauff übernommenen Aufgaben gehörte die Organisation der großen Migrationskongresse im Rahmen der Bemühungen, die Arbeit der ICMC und die Wanderungsprobleme ins internationale öffentliche Bewußtsein zu bringen. Diese Art der Öffentlichkeitsarbeit wurde in dem neuen Statut ausdrücklich hervorgehoben (Art. II/6). Nach Barcelona fand ein weiteres internationales Treffen 1954 in Breda in den Niederlanden statt, ein drittes 1957 in Assisi. Der Kongreß in den Niederlanden, der vom 11. bis zum 16. September stattfand und an dem 250 Personen teilnahmen, wurde organisatorisch und inhaltlich von Schauff in Zusammenarbeit mit den Direktor des Internationalen Katholischen Instituts für Sozialforschung in Den Haag, Professor G. H. Zeegers, vorbereitet. Diese Zusammenarbeit bewährte sich auch bei der Vorbereitung des Kongresses vom 22. bis zum 28. September 1957 in Assisi, auf dem Schauff über Europas Pflichten bei der Entwicklung Lateinamerikas referierte, nach seiner Auffassung eine Aufgabe von hoher Priorität ${ }^{56}$.

Im Kontext dieser Bemühungen, Flüchtlingspolitik aus christlicher und katholischer Verantwortung zu gestalten, stehen zeitlich und inhaltlich noch zwei besondere Projekte, die untrennbar mit dem Namen Schauff verbunden sind: Es sind dies einmal die Bemühungen um eine Lösung des Problems der Südtiroler, die für Deutschland optiert hatten und nach 1945 in ihre Heimat zurückgekehrt waren, zum anderen die Wiedergutmachung an den durch das nationalsozialistische Regime zur Zwangsarbeit nach Deutschland verschleppten Polen ${ }^{57}$.

53 Vgl. die Korrespondenz J. und Karin Schauff mit Pater Leiber, Juli/August 1956 (IfZ, NL Schauff, Bd. 14).

${ }^{54}$ Minutes Sixth Session ICMC, 12. 10. 1956 (NL Schauff, Bd. 15).

55 Ebenda.

56 Korrespondenz Schauff-Zeegers, 1954-1957 (IfZ, NL Schauff, Bd. 14); International Catholic Migration Congress, 1954. The Hague (Protokolle, Resolutionen - IfZ, NL Schauff, Bd. 29); Stark, Pionierjahre, in: Um der Freiheit willen, S. $221 \mathrm{ff.}$

57 Zum zweiten Problemkomplex siehe unten, S. $184 \mathrm{ff}$. 


\section{Die Südtiroler Irredenta}

Bereits während seiner Studienzeit war Schauff hinsichtlich des Problems Südtirol sensibilisiert worden. Er hatte u.a. in Berlin den Bozener Kanonikus Michael Gamper kennengelernt, einen Vorkämpfer für die Erhaltung der deutschen Kultur der Südtiroler gegen die Italienisierung. Im Kreis um Carl Sonnenschein war Schauff auch mit den sozialen Verhältnissen und Geistesströmungen in Italien vertraut geworden: Sonnenschein hatte während seiner römischen Studienzeit zum Kreis um Romolo Murri gehört, und der junge Schauff hatte von Sonnenscheins Wissen in diesem Bereich außerordentlich profitiert. Sonnenschein hatte $\mathrm{ihm}$ in Berlin zudem die Bekanntschaft von Luigi Don Sturzo vermittelt, der nach der Machtübernahme der Faschisten ins Exil gegangen war ${ }^{58}$. Auch den österreichischen Standpunkt in dieser Frage lernte Schauff durch einen Studienfreund kennen, der ebenfalls zum Kreis um Sonnenschein gehörte: Engelbert Dollfuß, der spätere österreichische Bundeskanzler.

Nach dem Krieg kam Schauff während seiner Tätigkeit für die ICMC sehr bald auch mit dem Problem der weiter bestehenden Südtiroler Irredenta in Berührung, zumal sich die Familie bei ihrer Rückwanderung nach Europa im Jahre 1949 nicht in Deutschland, sondern in Sterzing in Südtirol niederließ. Die Entscheidung für Südtirol, wo auch die Schauffschen Kinder zur Schule gehen sollten, war eine bewußte Option für das katholisch-lateinische Milieu, das die Eltern ja bereits im Rheinischen und dann in Lateinamerika geprägt hatte.

Zum Verständnis der Situation in Südtirol nach Kriegsende, schon seit 1918/19 eines der schwierigsten Minderheiten-Probleme in Europa, sind einige ausführlichere Anmerkungen notwendig. Die nationalsozialistische Volkstumspolitik hatte bekanntlich zwischen Ideologie und außenpolitischem Opportunismus geschwankt. Zwar wurden nach dem Nichtangriffspakt zwischen Hitler und Stalin und in dem darauffolgenden Krieg die Deutschen im Baltikum wie auch in Teilen Ost- und Südosteuropas "heim ins Reich" geholt oder in den von den Deutschen eroberten Gebieten angesiedelt ${ }^{59}$. Im Falle der deutschen Ethnie südlich des Brenners war allerdings das Interesse an einem Zusammengehen mit Italien vorrangig.

Bereits vor 1933 hatte Hitler für einen Verzicht auf Südtirol zugunsten des von ihm bewunderten Mussolini plädiert ${ }^{60}$. Nach der nationalsozialistischen Machtübernahme wurde die Anerkennung der Brennergrenze zum Preis für die Freundschaft Italiens, den Hitler und seine Paladine zu zahlen bereit waren. Vor allem im Zusammenhang mit dem Anschluß Österreichs und der Zerschlagung der Tschechoslowakei galt es in Rom Ängste zu zerstreuen, daß der Störfaktor Südtirol auf ähnliche Weise beseitigt werden könne.

In dieser Phase in der zweiten Hälfte der dreißiger Jahre wuchs die Idee, die Südtiroler en bloc ins Reich umzusiedeln, eine Lösung, die während des Ersten

58 Murri (1870-1944) und Don Sturzo (1871-1959) gehörten zu den Wegbereitern des politischen Katholizismus in Italien und der späteren Democrazia Cristiana. Don Sturzo war 1924-1946 im Exil in Paris, London und den USA.

59 Vgl. Schechtman, European Population Transfers, S. 485.

60 Hitler, Südtiroler Frage. 
Weltkriegs bereits von italienischer Seite erwogen worden war61. Ende April 1939 schließlich erfuhr eine Delegation von Südtirolern von Martin Bormann, was Himmler am 30. Mai 1939 offiziell bestätigen sollte: Südtirol sei als volksdeutsches Territorium aufzugeben - unter der Voraussetzung einer Umsiedlung von rund 200000 Südtirolern, die "deutsch sein wollen"62. Am 23. Juni 1939 wurde in Berlin ein entsprechendes Übereinkommen erreicht, dem bereits am 12. Oktober 1939 Richtlinien zur Durchführung des Projekts folgten. Sie stellten die Deutschen und Ladiner der Provinz Bozen sowie des 1926 davon abgetrennten Bozener Unterlandes, aber auch die übrigen deutschen Sprachinseln in den Provinzen Trient, Belluno und Udine vor die Alternative, bis zum 31. Dezember 1939 für die Auswanderung nach Deutschland oder das Verbleiben im Land als loyale italienische Staatsbürger ohne gesonderten Rechtsschutz zu optieren. Der Termin galt auch für die grundsätzlich zur Abwanderung verpflichteten deutschen Staatsbürger, von denen etwa 80 Prozent ehemalige Österreicher waren.

Der Assimilationsdruck der Italiener auf die Südtiroler war ohne jeden Zweifel ausgeprägt. Hinzu kam, daß den Südtirolern in zunehmendem Maße auch die wirtschaftliche Existenz erschwert wurde, wofür rigorose Enteignungsaktionen gegenüber den Bauern im Zusammenhang mit der Errichtung des Bozener Industriereviers ein anschauliches Beispiel lieferten ${ }^{63}$. Auf der anderen Seite gab es die massive, unter der Regie Himmlers und des Tiroler Gauleiters Franz Hofer durchgeführte nationalsozialistische Propaganda für eine „ethnische Flurbereinigung", die die Freiwilligkeit der schließlich erfolgten Option nachträglich in Frage stellen muß. Sicherlich hatten die Südtiroler nach 1933 voller Hoffnung auf Deutschland geblickt, und besonders für die Jugend war die nationalsozialistische Ideologie attraktiv geworden. Das mit dem „Stahlpakt" vom 23. November 1938 manifestierte deutsch-italienische Zusammengehen hatte jedoch alle Illusionen hinsichtlich einer „Rückkehr nach Deutschland“ zusammenbrechen lassen.

Die jetzt angebotene Lösung war sowohl von außen oktroyiert wie bitter enttäuschend. Corsini bezeichnet das Verfahren dieser Option als „Ungeheuerlichkeit und ein[en] Gewaltakt gegen die Geschichte“. Deren Wirklichkeit ergebe sich "aus der Verflechtung zwischen einem Land und den darin lebenden Menschen wenn es sich nicht um Nomaden handelt; diese Einheit bildet die Grundlage jeglicher Kultur und jeder Form kulturellen Wirkens. Diese Einheit zerbrechen, die Menschen aus einem Land herausnehmen, wo sie jahrhundertelang ansässig waren, ist keine historische Tat ..." 64 Der Verlust der Heimat wog schwer und führte dazu, daß keineswegs alle Südtiroler für eine Auswanderung stimmten - darunter so exponierte Persönlichkeiten des Volkstumskampfes wie der Kanonikus

61 Propagandisten in dieser Richtung waren Ettore Tolomei und Adriano Colocci-Vespucci. Vgl. Alexander/Lechner/Leidlmair, Heimatlos, S. 20.

62 Vgl. das entsprechende Dokument Himmlers in: Latour, Südtirol, S. 34-35.

63 Corsini/Lill, Südtirol, S. 243.

64 Ebenda, S. $323 \mathrm{f}$. 
Michael Gamper ${ }^{65}$ und der Senator Karl Tinzl ${ }^{66}$. Dennoch votierten etwa 80 Prozent der Optionsberechtigten in der Provinz Bozen für Deutschland ${ }^{67}$.

Dies war eine eindrucksvolle Mehrheit, deren Aussiedlung jedoch Schwierigkeiten bereitete. Dies betraf ebenso die von der Volksgruppe geforderte geschlossene Siedlung wie auch die zurückgelassenen Liegenschaften: Weder wurden nationalsozialistische Pläne realisiert, die Südtiroler wie andere volksdeutsche Gruppen in den eroberten Ostgebieten als „ethnisches Bollwerk" der „germanischen Rasse“ anzusiedeln, noch war das Reich in der Lage, diesen Menschen im Verlauf des fortschreitenden Krieges im Reich selbst eine neue Heimat zu geben. Von insgesamt rund 250000 Südtirolern und ca. 210000 Optanten gelangten etwa 75000 Menschen über den Brenner, von denen allerdings mehr als die Hälfte in Nordtirol blieb.

Allerdings kam die Umsiedlungsaktion schon bald ins Stocken und 1942/43 praktisch zum Erliegen. Gründe dafür waren die ungenügend geregelte Wohnungsfrage sowie das schwierige Problem der Arbeitsbeschaffung. Die Reichsbehörden waren vor allem an Volksdeutschen interessiert, die in der Industrie und beim Kriegseinsatz einsetzbar waren. Im Zuge des Kriegsverlaufs drängten zudem andere Probleme in den Vordergrund. Am 11. November 1943 wurde eine Sperre für Einbürgerungen aus Italien festgelegt, und nach dem Ende der faschistischen Herrschaft und dem Frontwechsel der Italiener blieb auch der politische Druck zur ethnischen Säuberung aus, während sich bei den zurückgebliebenen Südtirolern nunmehr wieder die Hoffnung regte, letzten Endes doch noch als Gebiet mit Deutschland vereint zu werden. Dazu trug die Besetzung Südtirols durch die Wehrmacht im Juli 1943 sowie die Einsetzung einer deutschen Verwaltung im September gleichen Jahres (Operationszone Alpenvorland) bei, die sich auf den vor Ort befindlichen deutschen Umsiedlungsapparat stützen konnten ${ }^{68}$. Karl Tinzl wurde anstelle des am 2. Dezember 1943 umgekommenen Peter Hofer zum Kommissarischen Präfekten der Provinz Bozen eingesetzt. Die Gelegenheit, tatsächlich Teil des deutschen Staatsgebiets zu werden, schien nie größer. Der Traum endete allerdings am 2. Mai 1945, als Südtirol von amerikanischen Truppen besetzt wurde.

Nach dem Ende des Zweiten Weltkriegs wurde zum zweiten Mal nach 1918 die Frage der Zugehörigkeit Südtirols zu Österreich oder Italien aufgeworfen. Die deutsche Volksgruppe südlich des Brenners sprach sich aufs neue unmißverständlich für den Anschluß an Österreich aus. Die Selbstbestimmung der Südtiroler wurde indessen wie nach dem Ersten Weltkrieg mißachtet; auch österreichische Vorstöße zur Wiedervereinigung der beiden Tiroler Landesteile stießen trotz anfänglicher Sympathien der westlichen Alliierten ${ }^{69}$ letzten Endes ins Leere. Am

65 Michael Gamper (1885-1956), Religionslehrer in Bozen. Eine Kurzbiographie in: Ermacora, Südtirol, S. 378-381.

66 Karl Tinzl (1888-1964), Rechtsanwalt in Bozen, ab 1921 Senator in Rom. Eine Kurzbiographie in: Ermacora, Südtirol, S. $381 \mathrm{f}$.

67 Die Zahlenangeben von deutscher und italienischer Seite sind unterschiedlich. Vgl. Corsini/Lill, Südtirol, S. 365-367; Alexander/Lechner/Leidlmair, Heimatlos, S. 25.

68 Ebenda, S. 44-49.

69 Ermacora, Südtirol, S. 42; Corsini/Lill, Südtirol, S. 468. 
24. Juni 1946 fiel dann die endgültige Entscheidung der vier Großmächte über die Aufrechterhaltung des Status quo in der Grenzfrage. An die Stelle des geforderten Selbstbestimmungsrechts trat die Südtiroler Autonomie.

Mehr Selbständigkeit sowohl in der Verwaltung als auch auf kultureller Ebene hatte die italienische Seite - vor allem Außenminister De Gasperi - den Alliierten bereits avisiert ${ }^{70}$. Im August $1946 \mathrm{kam}$ es zu einem ersten Treffen der italienischen und österreichischen Außenminister, De Gasperi und Gruber, im Rahmen der Friedensverhandlungen in Paris, das zu Ergebnissen führte, die Bestandteil des am 10. Februar 1947 abgeschlossenen Friedensvertrages zwischen Italien und den Alliierten wurden ${ }^{71}$. In dem Abkommen wurde noch einmal die italienische Grenzziehung am Brenner bestätigt, jedoch unter der Voraussetzung eines Autonomiestatuts für die Südtiroler Bevölkerung. Gefordert wurde aber auch die Wiedergutmachung der von Italien und dem Dritten Reich der Südtiroler Volksgruppe zugefügten Schäden; dazu gehörte in erster Linie eine Revision des Optionsgeschehens.

Die Optantenfrage spielte in den österreichisch-italienischen Verhandlungen eine ebenso große Rolle wie das Problem der Autonomie der Südtiroler Volksgruppe. Bei Kriegsende besaßen in Südtirol nur diejenigen die italienische Staatsbürgerschaft, die nicht für den Exodus optiert hatten; der Status der Optanten, die nicht abgewandert waren, war ebenso ungeklärt wie der der tatsächlich in Deutschland eingebürgerten Südtiroler. Die Regierung in Rom sprach allen Südtiroler Deutschlandoptanten die italienische Staatsbürgerschaft ab, eine Haltung, die vor allem von der Südtiroler Volkspartei (SVP) nach ihrer Gründung im Mai 1945 bekämpft wurde. Nach langem Tauziehen - in nationalistischen Kreisen Italiens wurde die Weiterführung der Aussiedlung propagiert ${ }^{72}$ - wurde schließlich von den Italienern im Friedensvertrag (Annex 4) die Revision der Optionen auch gegenüber den Vereinten Nationen anerkannt. Für die Südtiroler war die Rückführung der abgewanderten Landsleute um so wichtiger, als Italien nach Kriegsende schon bald wieder daranging, die italienische Einwanderung zu verstärken Österreich hatte in den Pariser Verhandlungen explizit darauf hingewiesen ${ }^{73}$. Auch ging man daran, ab 1947 Optanten, die im Lande geblieben waren, aus dem öffentlichen Dienst zu entlassen ${ }^{74}$.

Diese Tendenzen erforderten eine Nachbesserung und Präzisierung der in Paris getroffenen allgemeinen Vereinbarungen. Das Ergebnis war das zwischen der österreichisch-südtirolischen und der italienischen Seite ausgehandelte sogenannte Optantendekret vom 2. Februar 1948. Nachdem die SVP ihre Zustimmung zum Autonomiestatut gegeben hatte, konnte das „Gesetz zur Revision der Optionen der Südtiroler" verabschiedet werden und am 5. Februar 1948 in Kraft

70 So in dem Memorandum an den Außenministerrat vom 1.6. 1946. Corsini/Lill, Südtirol, S. 475-476.

71 Vgl. Rolf Steininger, Los von Rom?

72 Commissariato per le migrazione, Delegazione Alto Adige, Memorandum vom Juli 1945 an das italienische Innenministerium, zit. in: Alexander/Lechner/Leidlmair, Heimatlos, S. 194.

73 Memorandum an Botschafter Nicolò Carradini. Vgl. Toscano, Storia diplomatica, S. 366.

74 Alexander/Lechner/Leidlmair, Heimatlos, S. 208. 
$\operatorname{treten}^{75}$. Danach konnte die Option widerrufen und die italienische Staatsbürgerschaft zurückerworben werden; ausgeschlossen waren ehemalige aktive Nationalsozialisten und leitende Funktionäre der Auswanderungsstellen. Gesuche zur Rückoption von Personen, die sich in Italien bzw. Südtirol aufhielten, mußten innerhalb von drei Monaten erfolgen, von Personen außerhalb des Landes innerhalb von einem Jahr.

Das Ergebnis dieser Rückoptions-Aktion lag bei nahezu hundert Prozent ${ }^{76}$ blieb den Betroffenen doch nur die Wahl zwischen italienischer Staatsbürgerschaft und drohender Staatenlosigkeit. Die Rückbewegung wurde aber auch durch den Druck befördert, dem die in Österreich lebenden Südtiroler ausgesetzt waren; andererseits versuchten die Italiener, die Rückbewegung zu bremsen eben mit dem Argument, auf die Rückkehrer werde Druck ausgeübt. Auch bestand über den Passus in dem erwähnten Dekret, der "Nationalsozialisten“ von der Rückkehr ausschloß, die Möglichkeit, den Kreis unerwünschter Personen mehr oder minder beliebig auszuweiten 77 . Insgesamt wurde allerdings über 200000 Südtirolern, Optanten und ihren Familienangehörigen, die italienische Staatsbürgerschaft bestätigt bzw. wiederverliehen ${ }^{78}$.

Die reale Rückkehr in die Heimat gestaltet sich indessen schwierig. Dies betraf sowohl Wohnungsbeschaffung wie Arbeitsmöglichkeiten; Unsicherheiten bestanden aber auch hinsichtlich des Vermögentransfers sowie der Sicherung von Renten und Pensionen. Zudem zeigte sich in der Gesamtbilanz der Rückwanderung, daß die Mehrzahl der Umsiedler keineswegs zu den begüterten Schichten zählten. Schwerer als diese materiellen Sorgen wog indessen die seelische Irritation. Die Option wurde zum Trauma, den Rückwanderern haftete das Odium von Verrätern und Verlierern an.

Störend für eine rasche soziale Integration war vor allem ein Graben, der sich zwischen den ausgewanderten und den zurückgebliebenen Optanten auftat. Es wurden Vorwürfe des „Heimatverrats“ laut, und die mangelnde Hilfsbereitschaft gegenüber den Rückwanderern riß soziale Gräben auf. Zwar wurde im Frühjahr 1949 ein eigenes Fürsorgeamt für Rücksiedler eingerichtet, doch blieb die Rücksiedlungshilfe in Anbetracht der geringen finanziellen Mittel der Provinz und der Region äußerst beschränkt. Weder das Wohnungs- noch das Arbeitsbeschaffungsproblem konnte zufriedenstellend gelöst werden. Hinzu kam, daß das staatliche Arbeitsamt seine Aufgabe vor allem darin sah, den bis Anfang der fünfziger Jahre starken Zustrom von Arbeitskräften aus dem übrigen Italien unterzubringen.

Eine Reihe von Südtirolern war noch während und nach dem Kriege illegal nach Südtirol zurückgekehrt. Der erste geschlossene Rücksiedlertransport ge-

75 Decreto legislativo, 2. 2. 1948, veröffentlicht in: Gazzetta Uffiziale, 5. 2. 1948; Alexander/ Lechner/Leidlmair, Heimatlos, S. 214-216; Steininger, Südtirol, Bd. 1, S. $99 \mathrm{ff}$.

76 Alexander/Lechner/Leidlmair, Heimatlos, S. 223-227.

77 So erhielt der Mitbegründer des SVP, Karl Tinzl, erst 1953 die italienische Staatsbürgerschaft.

78 Italien und das Tiroler Etschland. Die Vereinbarung über die Revision der Optionen des Jahres 1933, in: Documenti. Berichte über das Leben in Italien, 21 (1957), S. 1594. 
langte erst am 10. Juni 1949 nach Bozen. Bis zum Jahre 1955 kehrten zwischen 20000 und 25000 Optanten in ihre Heimat zurück ${ }^{79}$.

Die Rückkehrer nach Südtirol sahen sich allerdings einer Fülle von wirtschaftlichen und sozialen Problemen gegenüber. Ihre Geldguthaben in Österreich und Deutschland wurden abgewertet ${ }^{80}$ und die von Umsiedlern angekauften Liegenschaften im Wege der Rückstellungsgesetzgebung meist entschädigungslos enteignet. In Südtirol selbst hatte die deutsche Umsiedlungs-Treuhand nach Kriegsende zahlreiche Liegenschaften an Italien übergeben müssen. In dieser Lage wog schwer, daß die zurückkehrenden Südtiroler Optanten weder von Österreich noch von Italien oder den alliierten Siegermächten als Flüchtlinge oder Vertriebene anerkannt oder ihnen gleichgestellt wurden. Daß diese Ausgrenzung schließlich doch durchbrochen werden konnte, geht einmal auf Eigeninitiativen der Südtiroler zurück. Von Gewicht waren in diesem Zusammenhang aber auch Initiativen und Hilfestellungen wie die von Johannes Schauff, der aufgrund eigener Erfahrung aktiv wurde und vor allem die noch junge deutsche Bundesrepublik und Italien zur Lösung des Südtirol-Problems zusammenzuspannen vermochte, das zu einem politischen Unruheherd mitten in Europa zu werden drohte.

Bei seiner Rückkehr nach Europa traf Schauff in Südtirol auf alte Bekannte wie den Kanonikus Gamper, der wieder an vorderster Front des Geschehens stand und ihm die Probleme auch kritisch zu vermitteln vermochte. Dies betraf neben der Optantenfrage die konkrete Ausgestaltung der zugesagten Autonomie. Hier wurde der Erlaß zu den Durchführungsbestimmungen des Pariser Vertrages jahrelang verzögert, der von den Südtirolern geforderte Sonderstatus der Provinz Bozen in der neuerrichteten Gesamtregion Trentino-Alto Adige wurde nicht gewährt.

Dem entsprach, daß es nach wie vor keine Gleichstellung der deutschen Sprache gab; dies bedeutete die Ausgrenzung von deutschsprachigen Südtirolern aus dem öffentlichen Dienst. Proteste Südtiroler Politiker wurden zum Teil von der italienischen Justiz strafrechtlich verfolgt, österreichische Politiker an der Grenze abgewiesen. 1960 brachte Österreich als faktische Schutzmacht das Südtirol-Problem vor die UNO, die sich jedoch nicht bereitfand, für eine Regionalautonomie der Provinz Bozen zu votieren. Vor Ort mündete die Entwicklung schließlich in schwere Unruhen und Attentate, die bereits in der zweiten Hälfte der fünfziger Jahre begonnen hatten. Sie zogen heftige Reaktionen der italienischen Behörden nach sich, worauf sich eine Spirale von Gewalt und Gegengewalt eröffnete, die die bilateralen Beziehungen Österreichs und Italiens jahrelang zu vergiften drohte ${ }^{81}$. Das Problem erhielt damit zusehends eine europäische Dimension.

79 Die genaue Rückkehrerzahl ist nicht zu eruieren (Alexander/Lechner/Leidlmair, Heimatlos, S. 246-249); Rudolf von Unterrichter nennt die Zahl von 25000 bis 30000 Rückkehrern (Unterrichter, Südtirol, S. 230).

80 Es handelte sich um ein Barvermögen von ca. 250 Millionen Reichsmark. Am 5. 7. 1950 wurde zwischen Italien und Österreich ein Abkommen über den Vermögenstransfer der Südtiroler Rücksiedler geschlossen, das allerdings durch die schlechten Wechselkurse und die Begrenzung der Transfersumme nur wenig Erleichterung zu schaffen vermochte. Vgl. Unterrichter, Südtirol, S. 231; Alexander/Lechner/Leidlmair, Heimatlos, S. 244.

81 Ein Beispiel ist die Pressekampagne um Folterungen in italienischen Polizei- und Carabinieristationen. Vgl. Ermacora, Südtirol, S. 97-123. 
Dies war die Lage, als sich Johannes Schauff des nach wie vor bestehenden Problems der Eingliederung der Optanten annahm und es bereits frühzeitig auf die Agenda der ICMC setzte, nachdem in Italien erneut Stimmen lautgeworden waren, diesen Menschen wieder die italienische Staatsbürgerschaft zu entziehen ${ }^{82}$. Um diese politischen Spannungen auflösen zu können, bedurfte es, wie Schauff wohl wußte, vielerlei Anstrengungen und die Ausnutzung von übergreifenden Kontakten zu beiden streitenden Parteien. Zunächst aber galt es, in Südtirol und Italien selbst das Terrain zu sondieren.

Über Don Sturzo hatte Schauff Alcide De Gasperi ${ }^{83}$ kennengelernt, der als italienischer Regierungschef und Außenminister ein gewichtiges Wort bei der Lösung der Südtirolfrage mitzureden hatte. Mit Mariano Rumor ${ }^{84}$ war er politisch und persönlich verbunden durch die gemeinsame Tätigkeit in der von Schauff mit initiierten „Féderation Internationale de Solidarité, einer Art christdemokratischer Internationale mit Aktionsschwerpunkt in Südamerika, auf die weiter unten noch einzugehen ist. In diesem Gremium gab es Kontakte mit weiteren Politikern der Democrazia Cristiana. Hier traf man sich aber auch mit deutschen christlichdemokratischen Politikern wie Heinrich Krone.

Für die Regelung des Südtirol-Problems vermochte Schauff weitere einflußreiche deutsche Politiker zu sensibilisieren, vor allem in Hinblick auf die mögliche Belastung der deutsch-italienischen Beziehung in bezug auf die von beiden Regierungen vorangetriebene europäische Integrationspolitik ${ }^{85}$. Um zum Abschluß der „Römischen Verträge" zu kommen, war die Beruhigung in Südtirol Conditio sine qua non. Diese Einsicht teilte vor allem auch der spätere Außenminister Heinrich von Brentano, mit dem Schauff und seine Familie nicht zuletzt durch das gemeinsame Engagement für eine Lösung der Südtiroler Irredenta eine tiefe Freundschaft verband ${ }^{86}$.

Daß die Bundesrepublik Deutschland - Österreich hatte auf entsprechende Vorstöße ablehnend reagiert - schließlich das Problem zu entschärfen suchte, indem sie die Südtiroler Optanten mit in die deutschen Lastenausgleichs-Maßnahmen einbezog, ist allerdings vor allem auf Initiativen von Südtirolern selbst zurückzuführen. $1961 \mathrm{nahm}$ der damalige Caritasdirektor der Diözese Brixen, Pfarrer Josef Zingerle, erste Verbindungen zu bundesdeutschen Behörden, insbesondere dem Bundesausgleichsamt auf. Der Bozener Rechtsanwalt Karl Tinzl führte zur gleichen Zeit flankierende Gespräche mit der römischen Regierung.

Bei der konkreten Umsetzung des Vorhabens, die Südtiroler Optanten in den deutschen Lastenausgleich einzubeziehen, spielte Johannes Schauff jedoch eine

82 Diese Forderung wurde von den DC-Senatoren Battaglia und Romano erhoben (Ermacora, Südtirol, S. 97).

83 Alcide De Gasperi (1881-1954), Democrazia Cristiana, mit Don Sturzo Begründer der Partito Populare im Widerstand gegen den Faschismus und die deutsche Besatzung; 19451953 Ministerpräsident und zeitweilig zugleich Außenminister; exponierter Europapolitiker, 1954 Präsident des Montan-Parlaments.

84 Mariano Rumor (1915-1990), Democrazia Cristiana, hatte zwischen 1959 und 1976 verschiedene Ministerämter inne und war 1968-70 und 1973-74 Ministerpräsident.

85 Die entsprechende Korrespondenz in IfZ, NL Schauff, Bd. 23.

$86 \mathrm{Vgl}$. Karin Schauff, Heinrich von Brentano, S. 47-52. 
wichtige Rolle. Dies gilt für die Bundesrepublik Deutschland, wo er er die Unterstützung vor allem der Abgeordneten Hermann Kopf, Wenzel Jaksch und Herbert Czaja sowie von Heinrich Krone gewann, der 1961 bis 1966 die Funktion eines Ministers für besondere Aufgaben wahrnahm, aber auch für Italien, wo Schauff auf die Unterstützung besonders des damaligen Ministerpräsidenten Giulio Andreotti bauen konnte.

Schauffs Aktivitäten in diesem Zusammenhang waren, wie es für ihn nicht untypisch ist, vor allem informeller Natur, deshalb jedoch nicht weniger wirkungsvoll. Dies zeigt z. B. sein Bericht an den deutschen Botschafter in Rom, Manfred Klaiber, über sein Treffen mit dem italienischen Parlamentsabgeordneten des Trentino, Alcide Berloffa, an dem auch Karl Tinzl teilnahm ${ }^{87}$. In diesem Memorandum berichtete Schauff über seine "Sondierungsmission im Rahmen der Lastenausgleichsleistungen für Südtiroler", eine Mission, die er in seiner Eigenschaft als Vertreter der ICMC und "als ein Freund des Bischofs Gargitter von Brixen“ habe durchführen können. Als Ergebnis seiner Sondierungsgespräche mit dem Auswärtigen Amt und dem Bundesfinanzministerium, die er ,in nicht amtlicher Funktion rein persönlich geführt" habe, kündigte er an, daß Berloffa mit dem Beratungsausschuß zusammenarbeiten ${ }^{88}$ und auch die Versorgung ehemaliger Südtiroler Wehrmachtsangehöriger unterstützen wolle. In einer ersten Besprechung habe er dem Abgeordneten „ein Promemoria“ „übergeben, in dem die Rechtslage der Entschädigungsleistungen sowie die in Aussicht genommene organisatorische Abwicklung " dargelegt sei ${ }^{89}$. Berloffa habe daraufhin Schauff vorgeschlagen, mit dem italienischen Botschafter in Bonn, Guidotti, in Verbindung zu treten und „ihn im Sinne des von mir Berloffa übergebenen Memorandum zu unterrichten." Er habe ihm ein Empfehlungsschreiben an den Botschafter mitgegeben, da von ihm „die Zustimmung der italienischen Behörden weitgehend ... abhänge".

Schauff kündigte Klaiber weiter an, er werde in Bonn mit Guidotti Kontakt aufnehmen ${ }^{90}$, jedoch vorher noch das Auswärtige Amt sowie das Bundesfinanzministerium über das „Ergebnis meiner römischen Gespräche informieren“. Das Schauffsche „Pro Memoria“ über die Südtirolfrage wurde schließlich zu einer Diskussionsgrundlage sowohl im Bundesfinanzministerium wie auch für entsprechende Ressortbesprechungen im Auswärtigen Amt ${ }^{91}$. Mit der Einbeziehung der

87 Das Schreiben vom 4. 6. 1963, in: PAAA, B 24, Nr. 487/3 (Bestand Nationale Minderheiten/Südtirol). Der Kontakt mit Berloffa war offenbar durch Vermittlung von Rudolf v. Unterrichter zustandegekommen. Vgl. das Schreiben Unterrichters an Schauff vom 20. 7. 1983 (IfZ, NL Schauff, Bd. 39).

88 Berloffa wurde nach dessen Gründung 1964 in den Beratungsausschuß für Umsiedlungsgeschädigte berufen. Vgl. Unterrichter, Südtirol, S. 238.

89 Das Memorandum in IfZ, NL Schauff, Bd. 39.

90 Gastone Guidotti war ab 1958 italienischer Botschafter in Wien. Er hatte auf italienischer Seite als einer der ersten das Südtirol-Problem erkannt, das zu einer langfristigen schweren Belastung der italienischen Außenpolitik werden konnte. Er konnte sich jedoch mit seinen Vorstellungen, das Problem vor den Internationalen Gerichtshof im Haag zu bringen, in Rom nicht durchsetzen. Vgl. Steininger, Südtirol, Bd. 2, S. $189 \mathrm{ff}$.

91 Vgl. Notiz Dr. Sauter im Bundesfinanzministerium über eine Besprechung mit Schauff vom 12.7. 1963 (eine erste Besprechung fand am 8.7. und eine weitere Ressortbespre- 
Südtiroler in die 14. Lastenausgleichsnovelle ${ }^{92}$ und später durch verschiedene bilaterale deutsch-italienische Abkommen konnten die schwierigsten sozialen und materiellen Probleme entschärft werden, die durch die Option entstanden waren. Diese betraf u. a die Altersversorgung, die Gewährung von Darlehen sowie Hausratsbeihilfen. Solche Unterstützungsmaßnahmen konnten jedoch nur dann effektiv durchgeführt werden, wenn die einzelnen Fälle vor Ort auf ihre rechtlichen, wirtschaftlichen und sozialen Voraussetzungen geprüft wurden. Das für Italien zuständige Ausgleichsamt Köln besaß dazu allein nicht die Kapazität. Deshalb wurde nach einer Übereinkunft von Auswärtigem Amt und Bundesfinanzministerium mit der italienischen Regierung und durch Erlaß des Präsidenten des Bundesausgleichsamts am 16. Juni 1964 in Bozen ein ehrenamtlicher „Beratungsausschuß für Umsiedlungsgeschädigte" eingerichtet, der die organisatorisch-bürokratische Umsetzung der vorgesehenen Entschädigungsmaßnahmen mit übernehmen sollte. Ihm gehörten eine repräsentative Auswahl von Persönlichkeiten aus Südtirol und von italienischer Seite an - darunter Vertreter der Sozialverbände, der Optanten, der Kirche und des öffentlichen Lebens ${ }^{93}$. Den Vorsitz übernahm auf Wunsch der betroffenen Südtiroler wie auch der zuständigen deutschen Bundesbehörden ${ }^{94}$ Johannes Schauff, Geschäftsführer wurde der gebürtige Brixener und ehemalige Optant Rudolf von Unterrichter.

Aufgabe des neuen Gremiums war vor allem die Feststellung und Erhebung der Schäden bei annähernd 20000 Personen. Dies betraf die schwierige Betriebs- und Bodenbewertung auf dem Stand von 1938, die nach Einzelantrag und auf der Grundlage individueller Nachweise vorgenommen werden mußte, die oft schwierige Suche nach Erben, die Feststellung während der Umsiedlung transferierter Barvermögenswerte und anderes mehr ${ }^{95}$. Mit seiner Tätigkeit erfüllte der Beratungsausschuß eine Mittlerfunktion zwischen der Bonner Regierung, dem Präsidenten des Bundesausgleichsamtes und den beteiligten Banken und bildete auf der anderen Seite eine notwendige Brücke zur italienischen Regierung sowie den italienischen Behörden in Rom, Trient und Bozen. Vor allem letzteres war die Auf-

chung am 10.7. 1963 statt): „Das Bundesministerium für Finanzen wird nunmehr an das Auswärtige Amt mit der schriftlichen Bitte herantreten, die italienische Regierung offiziell über die 14. Novelle zum LAG und für die von uns vorgesehenen Vorkehrungen zur Durchführung des Gesetzes zu unterrichten." Die Kontaktierung des Auswärtigen Amts durch das BMF ist in einem Vermerk vom 7.9. 1963 festgehalten; die Diskussionsgrundlage für die Ressortbesprechung im Auswärtigen Amt sei das Schauffsche „Pro Memoria“ gewesen (PAAA, V 7 (507) - 82.03/3).

92 Das Lastenausgleichsgesetz trat 1961 in Kraft und regelte die Gewährung von Beihilfen an Vertriebene im Ausland; es wurde 1963 im Hinblick auf Berücksichtigung von Südtiroler Umsiedlungsgeschädigten und Optanten erweitert.

93 Die Zusammensetzung des Beratungsausschusses (Namensliste) bei: Unterrichter, Sütirol, S. 238.

94 Schauff berichtet in einem Brief an das Deutsche Generalkonsulat in Mailand (Dr. Rumpf) vom 20. 3. 1964, daß ihn die „Bundesregierung “ gebeten habe, den Vorsitz des Beratungsausschusses zu übernehmen; eine gleiche rückblickende Aussage in einem Brief Schauffs an Bundeskanzler Helmut Kohl vom 9.9. 1983 (beide Schreiben in IfZ, NL Schauff, Bd. 23).

95 Vgl. Unterrichter, Südtirol, S. $234 \mathrm{ff}$. 
gabe des Vorsitzenden Johannes Schauff, der „seine bestehenden guten Verbindungen in Rom und Bonn" zu nutzen verstand ${ }^{96}$. Ein enges Kooperationsverhältnis mit konkreter Aufgabenteilung entwickelte sich mit dem Bundesausgleichsamt und dessen Präsidenten Karl Heinz Schaefer, der Ende 1984 als Nachfolger von Schauff den Vorsitz des „Beratungsausschusses für Umsiedlungsgeschädigte“ übernehmen sollte ${ }^{97}$.

Neben der Gewährung umfangreicher Darlehen an Optanten durch die Lastenausgleichsbank bzw. die Deutsche Landesrenten- und Siedlungsbank ${ }^{98}$ erreichte der Beratungsausschuß am 14. Januar 1967 auch eine Vereinbarung zur Rückgabe des noch nicht verkauften Optantenbesitzes in der Provinz Trient, der sich als Feindeigentum unter italienischer Treuhandverwaltung befand ${ }^{99}$. Im gleichen Jahr erfolgte auch die Einbeziehung Südtiroler Optanten in das deutsch-italienische Abkommen über die Regelung von Kriegsschäden vom 19. Oktober 1967. Schließlich wurden die Optanten mit der Einbeziehung in das deutsche Reparationsgesetz vom 25. Februar 1969 den Geschädigten der Bundesrepublik gleichgestellt.

Auch die Lösung eines weiteren Problems ging wesentlich auf Schauffs Initiative zurück: Infolge der Option war die Mehrzahl der in deutschen Dienststellen oder in den Ansiedlungsgebieten beschäftigten Südtiroler in den Jahren 1939 bis 1948 ohne Pflichtversicherung geblieben - nach der Rückkehr aufgrund der erheblichen materiellen Konsequenzen ein Gegenstand großer Erbitterung, die sich vor allem gegen Rom richtete. Nach über zehnjährigen Verhandlungen trat am 27. Januar 1976 ein deutsch-italienisches Abkommen in Kraft, das die Frage der Nachversicherung regelte. Dem Beratungsauschuß für Umsiedlungsgeschädigte kam dabei die Aufgabe zu, als Voraussetzung für die Gewährung zusätzlicher oder rechtsbegründeter Rentenzeiten die entsprechenden verbindlichen Bescheinigungen auszustellen.

Der „Beratungsausschuß für Umsiedlungsgeschädigte“ beendete seine Tätigkeit im Juni 1999. Bis dahin waren in den 35 Jahren seit seiner Gründung rund 121,3 Millionen DM an sozialen Leistungen, Entschädigungszahlungen und günstigen Krediten ausgezahlt worden. Darüber hinaus gewährte die Bundesrepublik Deutschland als Rechtsnachfolgerin des Deutschen Reichs für die in der Optionszeit ausgefallenen Rentenjahre weitere 262 Millionen DM. Rund 20000 Umsiedler

96 So Schaefer in: Der Beratungsauschuß, S. 3; Unterrichter berichtet, daß Schauff „nicht nur die mit den verschiedenen Ressorts in Bonn erforderlichen Gespräche" vorbereitet, sondern auch "die noch weit schwierigere Koordination mit den römischen Zentralstellen" übernommen habe (Unterrichter, Südtirol, S. 234f.).

97 Schaefer war ab 1975 Präsident des Bundesausgleichsamtes; er war bereits als Vizepräsident dieser Behörde seit Beginn der sechziger Jahre, d.h. seit Beginn der Versuche, das Problem der Südtiroler Optanten durch Einschaltung der Bundesregierung zu lösen (siehe oben, S. 118 ff.), mit dem Optanten-Problem befaßt.

98 Die Kapital- und Finanzierungsoperationen sowie die Darlehensverwaltung wurden vor Ort durch die Volksbank Brixen (heute Südtiroler Volksbank) abgewickelt.

$99 \mathrm{Zu}$ diesen Restitutionsverhandlungen auch im Bereich der Sozialversicherung vgl. Unterrichter, Lo status, S. 145-223 (mit Vertragstexten im Anhang). 
beziehungsweise Optanten kamen in den Genuß dieser Zahlungen ${ }^{100}$. Auf der Abschlußveranstaltung des Beratungsausschusses am 4. Juni 1999 in Bozen würdigte der letzte amtierende Vorsitzende Karl Heinz Schaefer die Rolle seines Vorgängers: „Ich sehe es als Erfolg der hartnäckigen, von Dr. Johannes Schauff unterstützten südtiroler Bemühungen, daß sich Bundesregierung und Parlament zu deutschen humanitären Leistungen, später auch zu Entschädigungen bereitfanden."101

Schauffs Engagement für die Südtiroler Sache dokumentiert ein anderer Vorgang, der die deutsch-italienischen und südtirolisch-italienischen Beziehungen belastete. Dabei handelt es sich um das Attentat italienischer Partisanen auf ein deutsches Polizeibataillon am 23. März 1944 in der Via Rasella in Rom. Betroffen waren Angehörige der 11. Kompanie des 3. Bataillons des Polizeiregiments „Bozen“, das nach der Einrichtung der „Operationszone Alpenvorland“ aus Südtirolern gebildet worden war ${ }^{102}$. Das von der kommunistischen Widerstandsorganisation "Gruppi d'Azione Patriotica" verübte Attentat war Teil der Strategie, deutsche Repressalien gegenüber der Zivilbevölkerung in der „offenen Stadt “ zu provozieren, um damit die Passivität der Bevölkerung zu überwinden und die Motivation zum Widerstand zu stärken. Bei dem Anschlag kamen 27 Polizisten sofort ums Leben, sieben weitere erlagen am selben Abend und am Tag darauf im Feldlazarett bzw. Krankenhaus ihren Verletzungen. Weitere 45 Männer wurden so schwer verletzt, daß sie als Verlust galten; es gab darüber hinaus zahlreiche verwundete Zivilisten ${ }^{103}$.

Im Zuge der deutschen Repressalien wurden am 24. März 1944 in den Fosse Ardeantine 335 italienische Geiseln erschossen ${ }^{104}$. Für diese Exekutionen sollte zunächst auch die betroffene Polizeieinheit herangezogen werden. Von diesem Vorhaben nahm die deutsche Führung aber wieder Abstand: Man befürchtete offenbar angesichts der starken katholischen Prägung der Südtiroler ${ }^{105}$ unter Umständen Widerstand ${ }^{106}$.

Nach dem Kriege wurde den Angehörigen der Attentatsopfer mit dem Argument, es habe sich um SS-Einheiten gehandelt, jede Entschädigung verweigert. Auch in späteren historischen Abhandlungen dieses Geschehens wurde diese Behauptung immer wieder vorgebracht und den betroffenen Südtirolern sogar unterstellt, sie hätten sich bei der Bekämpfung der Partisanen durch besondere Brutalität hervorgetan ${ }^{107}$.

Gegen diese Behauptung, die unterdessen auch durch die Forschung widerlegt ist ${ }^{108}$, zog Johannes Schauff aufgrund eigener Recherchen engagiert zu Felde ${ }^{109}$ :

100 Zahlengaben nach Schaefer, Der Beratungsausschuß, S. 6 ff.; Interview mit Schaefer in Dolomiten, 5./6. 6. 1999, S. 17.

101 Schaefer, Beratungsausschuß, S. 2 f.

102 Vgl. Hartungen, Polizeiregimenter, S. 494-516.

103 Zahlenangaben bei Prauser, Geiselerschießungen, S. 45.

$104 \mathrm{Zu}$ dem Vorgang Prauser, ebenda, als bislang neuester Forschungsstand.

105 Bei den Attentatsopfern hatten sich zahlreiche Rosenkränze gefunden.

106 Vgl. Prauser, ebenda, S. 75 ff., 82.

107 So Katz, Mord in Rom, S. 39 f.; Hartungen, Polizeiregimenter, S. 509.

108 Prauser, Geiselerschießung, S. 49 f. Ein abschließend bilanzierender Aufsatz von Steffen Prauser erscheint 2001 in den VfZ. 
Die meisten der zu diesem Polizeiregiment zwangsweise einberufenen Männer, darunter auch Südtiroler, die für Italien optiert hatten, waren Familienväter im Durchschnittsalter von 35 bis 36 Jahren ${ }^{110}$ und hatten keinerlei Kampferfahrung. Es handelte sich lediglich um ein Hilfskorps, das keinerlei Kennzeichen der SS getragen und dessen Offiziere die bei der Polizei üblichen Dienstgradbezeichnungen geführt hatten ${ }^{111}$.

Angesichts der Tatsache, daß die gesamte Polizei seit 1935 Himmler unterstellt war, unterstand die Einheit zwar nominell dessen Beauftragten in Norditalien, SSObergruppenführer und General der Waffen-SS Karl Wolff, in Rom jedoch dem Stadtkommandanten der Wehrmacht. Die Einheit war zu Wachdiensten am Quirinal eingeteilt und marschierte täglich, vom Foro Mussolini kommend, wo Schießübungen zu absolvieren waren, über die Piazza del Popolo,Via del Babuino, Via dei Due Macelli, Piazza di Spagna, Largo del Tritone und durch die Via Rasella zu ihren Unterkünften, die im Castro Pretorio im Quirinal lagen.

Schauffs Kampf um eine Rehabilitierung dieser doppelt gestraften Personengruppe war kein materieller Erfolg beschieden; er hatte vor allem eine moralische Dimension, die half, viele Wunden zu heilen, und trug maßgeblich zu dem hohen Ansehen bei, das Johannes Schauff insbesondere in Südtirol genoß.

109 Vgl. die Korrespondenz mit P. Robert Graham SJ (La Civiltà Cattolica), Graf Bossi Fedregotti und Hugo Gamper/Bozen; Informierung von Landeshauptmann Magnago und Staatspräsident Leone; Pressenotizen und öffentliche Auseinandersetzung mit dem 1967 erschienenen Buch von Robert Katz „Death in Rome“ (IfZ, NL Schauff, Bd. 3 und 24); Schauff, Autobiographische Notizen/Fragmente.

110 Ein soziales und biographisches Profil der Gruppe findet sich bei Prauser, Geiselerschießung, S. $45 \mathrm{ff}$.

11 Das Polizeiregiment Bozen wurde erst mit dem Erlaß vom 16. 4. 1944 wie die deutschen Pendants als „SS-Polizeiregiment“ bezeichnet. Vgl. Neufeldt/Huck/Tessin, Ordnungspolizei, S. 286; Goetz, Attentat, S. $166 \mathrm{ff}$. (mit weiterführender Literatur, vor allem auch von italienischer Seite). 